\title{
Experiencias en la medida del valor agua-cemento según el procedimiento Thaulow

\section{INTRODUCCION}

En la nueva DIN 1045 se ha mencionado por vez primera en una norma alemana del hormigón armado el valor ( $\mathrm{W} / \mathrm{Z}$ ó a/c) como concepto y se ha introducido como requisito de calidad del hormigón con especiales propiedades. Como el cemento necesita para su elaboración más agua que para el fraguado y el endurecimiento, una parte del "agua excedente" se evapora y deja poros que influyen negativamente en la densidad de la pasta de cemento y, por tanto, en la resistencia y en otras propiedades del hormigón.

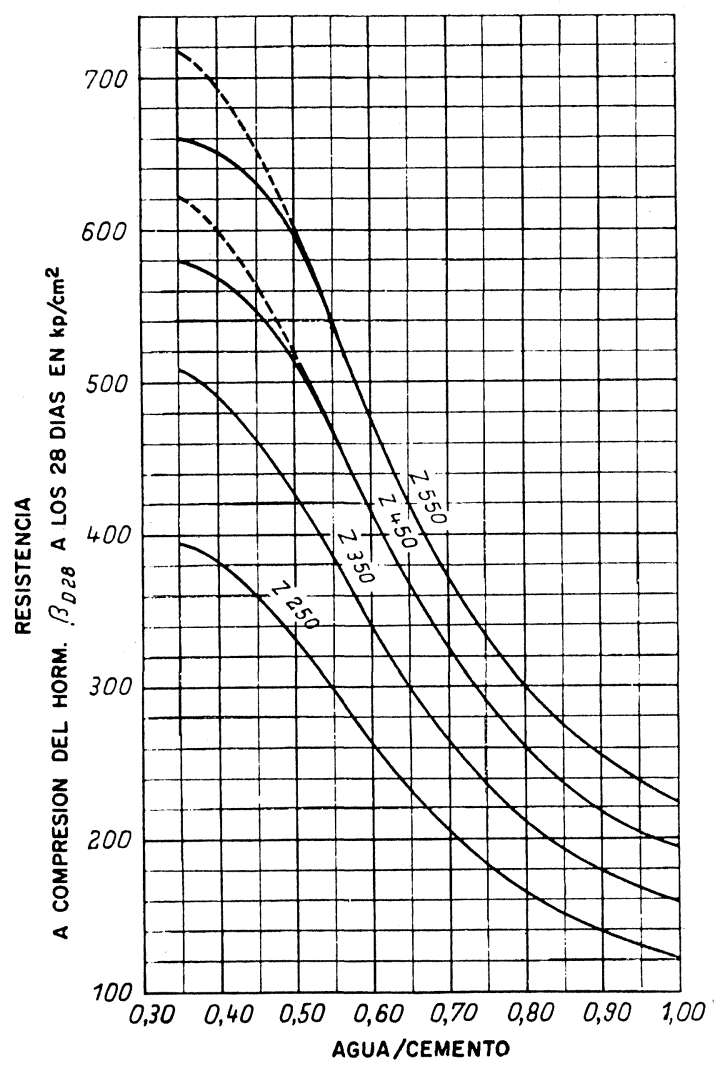

Según la ley de Abrams (1) se eliminan por ello mutuamente las influencias del volumen de cemento y agua en el hormigón, de manera que la resistencia a compresión del hormigón endurecido depende exclusivamente del valor a/c si las demás circunstancias se mantienen idénticas. La figura 1 muestra la relación conncida [según (2)]. Esto es lo que rige respecto de las nuevas normas y sustituye al diagrama empleado hasta ahora [según (3); ver también figura 2].

Los tecnólogos del hormigón han considerado siempre como defecto el que la resistencia del hormigón sólo pueda conocerse en general al cabo de los 28 días, y en casos especiales quizás al cabo de 7 días. Una reacción tan "prolongada" no es adecuada para controlar la producción de hormigón. Se ha ido imponiendo por ello, cada vez más, en el de- 
cenio pasado la idea de que la calidad del hormigón debe controlarse mediante el valor a/c. Thaulow (4) propuso en su sistema de control del hormigón fresco un procedimiento muy sencillo para la evaluación del valor a/c. Desde hace más de 12 años, el autor ha tenido la oportunidad de comprobar el procedimiento de Thaulow en la medida del valor a/c en el control de calidad de las obras y en las centrales de hormigón. Un ejemplo práctico, según las medidas del autor en un lugar de obra $\left(15.000 \mathrm{~m}^{3}\right.$ de hormigón), pondrá de manifiesto la idoneidad de la evaluación del a/c para controlar la calidad.

Durante la fabricación del hormigón se midió el valor a/c del hormigón fresco, aproximadamente cada media hora. De la calidad de hormigón B 225 se obtuvieron 1.179 valores. El mismo hormigón fue sometido también a un ensayo de calidad según DIN 1045. Para ello se ensayaron 158 juegos de cubos, cuya producción se distribuyó casi homogéneamente a lo largo del tiempo de hormigonado. Los resultados de los ensayos del laboratorio de la planta de suministro informaron sobre la resistencia normalizada del cemento empleado. Fueron aproximadamente 200 resultados. La resistencia normalizada de cemento no fue menor de $500 \mathrm{kp} / \mathrm{cm}^{2}$. La figura 2 muestra la conocida relación entre la resistencia del hormigón, valor a/c y resistencia del cemento según Walz. En el eje del a/c se registró el diafragma de frecuencia de los valores a/c medidos hacia abajo. Si esta distribución se lleva por encima de la ordenada en la curva de la resistencia del cemento, se obtiene en ésta la distribución de la resistencia de los cubos que es de esperar. Para establecer la comparación se registró igualmente en la ordenada la distribución de las resistencias de los cubos realmente medidas. Se reconoce que la coincidencia es perfecta.

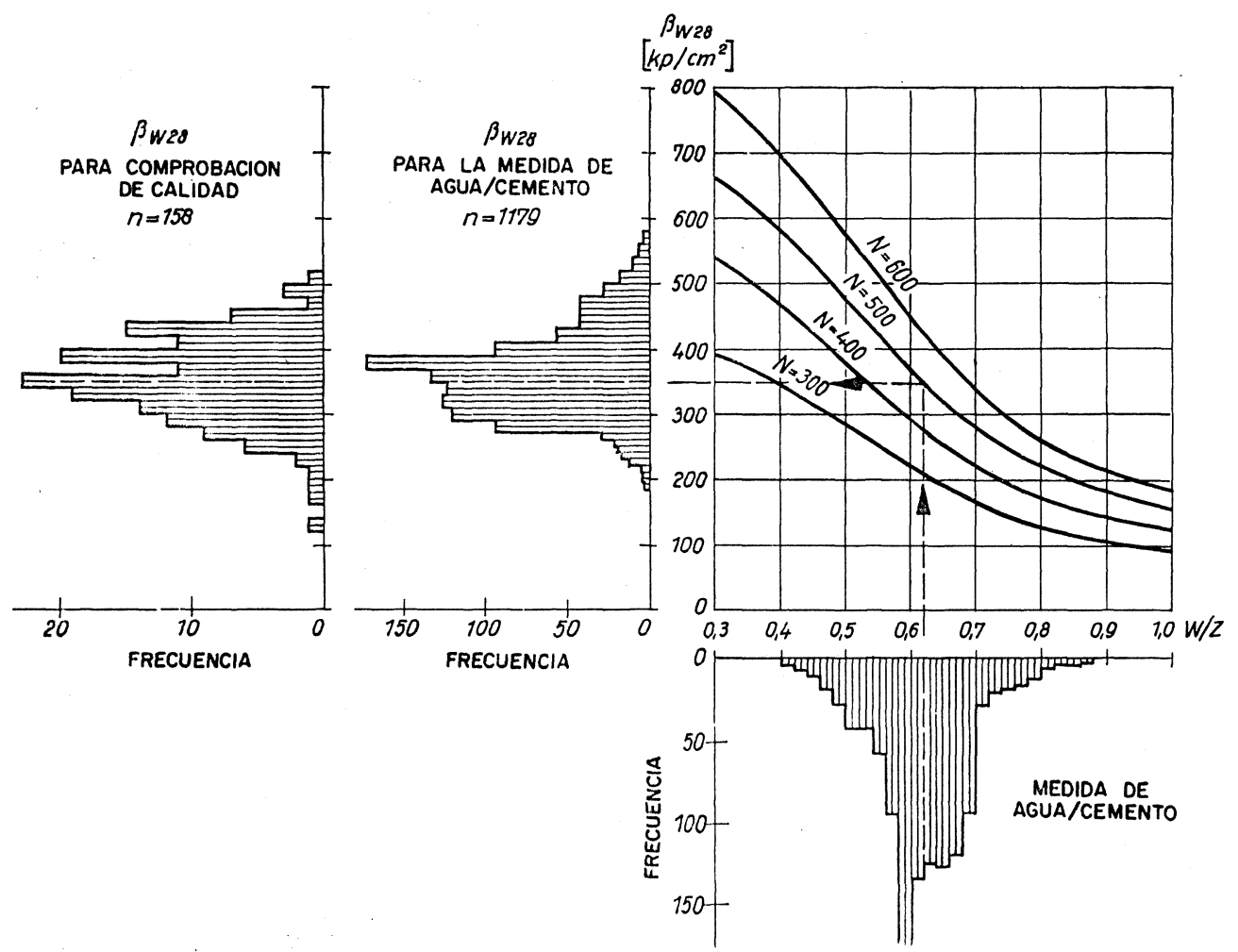

Fig. 2.-Partiendo de la distribución de frecuencia del valor a/c medida $y$ con ayuda de la relación entre el valor de a/c y la resistencia del hormigón se puede deducir la distribución de la resistencia que se puede esperar. Los resultados de las medidas de a/c presentan una perfecta coincidencia con la distribución de resistencias en los cubós de comprobación de calidad.

Es, por tanto, posible ya durante la fabricación del hormigón obtener una información sobre la resistencia que se puede esperar y, por consiguiente, controlar inmediatamente la calidad. 
En DIN 1045 se autorizó, por vez primera, aun cuando con restricciones, la evaluación del valor a/c en lugar de la comprobación por cubos.

En los siguientes apartados se describe el procedimiento de Thaulow, así como se deriva y se indica un diagrama que simplifica y facilita en gran manera los cálculos relacionados con la medida del valor a/c. En el apartado 4 se comunican finalmente algunos resultados de las evaluaciones del valor a/c, que proporcionarán una impresión acerca de la distribución del valor a/c en los lugares de obras y en las fábricas de hormigón. En el apartado 5 se indicarán, junto a un resumen, otras evoluciones que han resultado de las experiencias en la medida del valor a/c.

\section{PROCEDIMIENTO DE THAULOW}

El procedimiento propuesto por Thaulow (4) para la evaluación del valor a/c, parte de las siguientes consideraciones:

El peso debajo del agua de una muestra de hormigón fresco sin aire debe ser igual a la suma de los pesos subacuáticos de cada una de las componentes del hormigón fresco:

$$
G_{0}^{\prime}=Z_{0}^{\prime}+K_{0}^{\prime}+W^{\prime}{ }_{0}
$$

El peso subacuático del agua, como se sabe, es cero. Los pesos subacuáticos del cemento y áridos resultan:

$$
\begin{aligned}
& Z_{0}^{\prime}=\frac{s_{\mathrm{z}}-1}{s_{\mathrm{z}}} \cdot \mathrm{Z} ; \\
& \mathrm{K}_{0}^{\prime}=\frac{s_{\mathrm{k}}-1}{s_{\mathrm{k}}} \cdot \mathrm{K} .
\end{aligned}
$$

Entonces tenemos:

$$
G_{0}^{\prime}=\frac{s_{\mathrm{z}}-1}{s_{\mathrm{z}}} \cdot Z+\frac{s_{\mathrm{k}}-1}{s_{\mathrm{k}}} \cdot K
$$

Si se introduce la proporción de mezcla $k=K / Z$ :

$$
\begin{gathered}
G_{0}, Z=\frac{s_{\mathrm{z}}-1}{s_{\mathrm{z}}}+\frac{s_{\mathrm{k}}-1}{s_{\mathrm{k}}} \cdot k ; \\
\left(\frac{s_{\mathrm{z}}-1}{s_{\mathrm{z}}}+\frac{s_{\mathrm{k}}-1}{s_{\mathrm{k}}} \cdot k\right)
\end{gathered}
$$

El peso de la muestra de hormigón fresco se compone de los pesos de cada una de la componentes:

$$
\begin{aligned}
G & =Z+K+W ; \\
G / Z & =1+k+W / Z ; \\
W / Z & =G / Z-(1+k) .
\end{aligned}
$$

Con $Z$ de la ecuación [6] resulta:

$$
W / Z=\frac{G}{G_{0}^{\prime}}\left(\frac{s_{\mathrm{z}}-1}{s_{\mathrm{z}}}+\frac{s_{\mathrm{k}}-1}{s_{\mathrm{k}}} \cdot k\right)-(1+k) .
$$


En esta fórmula significan:

$G=$ Peso de la muestra de hormigón fresco (normal $10 \mathrm{~kg}$ ).

$G_{0}^{\prime}=$ Peso de la muestra de hormigón fresco sin aire bajo el agua.

$s_{\mathrm{z}}=$ Densidad pura del cemento (normal $3,1 \mathrm{~kg} / \mathrm{l}$ ).

$s_{\mathrm{k}}=$ Densidad pura de los áridos (normal 2,60 a $2,70 \mathrm{~kg} / \mathrm{l}$ ).

$k=K / Z=$ relación de los áridos: cemento (gravimetría).

El valor $G_{0}^{\prime}$ se mide según el procedimiento Thaulow. La hoja de trabajo para la comprobación del hormigón fresco contiene una amplia descripción del procedimiento de ensayo. Un extracto se ha reproducido en la figura 3 . La realización se halla representada en la figura 4.

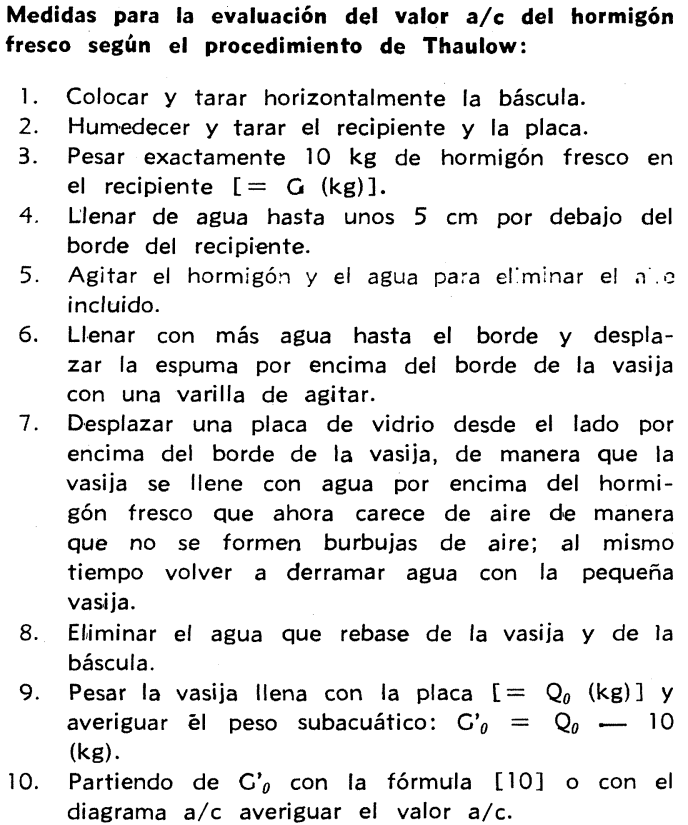

4. Lilenar de agua hasta unos $5 \mathrm{~cm}$ por debajo del borde del recipiente.

5. Agitar el hormigón y el agua para eliminar el aia incluido.

6. Llenar con más agua hasta el borde y desplazar la espuma por encima del borde de la vasija con una varilla de agitar.

7. Desplazar una placa de vidrio desde el lado por encima del borde de la vasija, de manera que la vasija se llene con agua por encima del hormigón fresco que ahora carece de aire de manera que no se formen burbujas de aire; al mismo tiempo volver a derramar agua con la pequeña vasija.

8. Eliminar el agua que rebase de la vasija y de la báscula.

9. Pesar la vasija llena con la placa $\left[=Q_{0}(\mathrm{~kg})\right]$ y averiguar èl peso subacuático: $\mathrm{C}_{0}^{\prime}=\mathrm{Q}_{0}-10$ ( $\mathrm{kg})$.

10. Partiendo de $\mathrm{C}_{0}^{\prime}$ con la fórmula [10] o con el diagrama a/c averiguar el valor $a / c$

Fig. 3.-Extracto de la hoja de trabajo para el ensayo de hormigón fresco según el procedimiento de Thaulow (5).

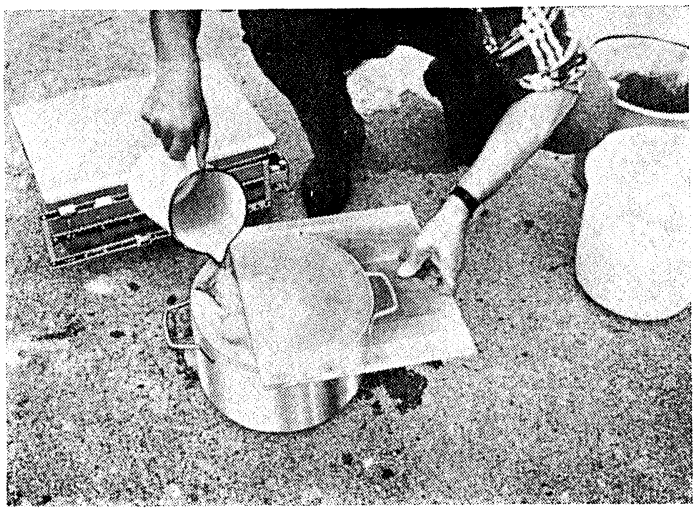

Fig. 4.-Comprobación del hormigón fresco según e procedimiento de Thaulow.

Las densidades puras de cemento y áridos son constantes en general respecto de un determinado lugar de obras; sólo deben medirse una vez. Esto puede suceder asimismo por ejemplo con el procedimiento de Thaulow según la hoja de trabajo para la comprobación de áridos (6).

La relación de mezcla $k=K / Z$ se obtiene de las lecturas de la báscula de la instalación de mezclado de hormigón.

Se puede evitar el cálculo por la ecuación [10], si se utiliza una "reserva de cálculo" en forma de un diagrama cuidadosamente averiguado (ver apartado 3). 


\section{EL DIAGRAMA a/c}

Al evaluar la ecuación [10] se sabe que el valor a/c se puede calcular como diferencia de dos valores casi iguales. El resultado es muy sensible al error, la exactitud de la regla de cálculo no basta muchas veces. Por ello es conveniente efectuar el cálculo en forma de un diagrama de una vez por todas. La ecuación [10] se transforma como sigue:

$$
\begin{gathered}
\mathrm{W} / \mathrm{Z}=\frac{G}{G_{0}^{\prime}} \cdot \frac{s_{\mathrm{z}}-1}{s_{\mathrm{z}}}+\frac{G}{G_{0}^{\prime}} \cdot \frac{s_{\mathrm{k}}-1}{s_{\mathrm{k}}} \cdot k-1-k . \\
\mathrm{W} / \mathrm{Z}=\left(\frac{G}{G_{0}^{\prime}} \cdot \frac{s_{\mathrm{z}}-1}{s_{\mathrm{z}}}-1\right)+k \cdot\left(\frac{G}{G_{0}^{\prime}} \cdot \frac{s_{\mathrm{k}}-1}{s_{\mathrm{k}}}-1\right) \\
\mathrm{W} / \mathrm{Z}=A+k \cdot B .
\end{gathered}
$$

Como $G, s_{\mathrm{k}}$ y $s_{\mathrm{z}}$ son parámetros conocidos, los miembros $A=f_{\mathrm{a}}\left(G_{0}^{\prime}\right)$ y $B=f_{\mathrm{b}}\left(G_{0}^{\prime}\right)$ representan hipérbolas, que pueden representarse en el cuadrante izquierdo y derecho superior del diagrama de la figura 5 . Los ejes $A$ o $B$ marchan hacia la izquierda o derecha en forma positiva.

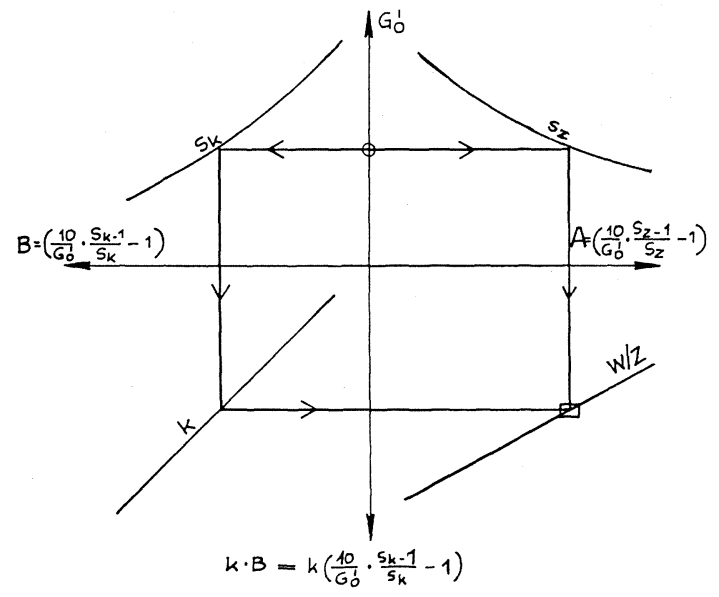

Fig. 5.-Representación gráfica de la ecuación [12] como base del diagrama de $a / c$ (ver figura 6 ).

En el cuadrante inferior izquierdo se ha efectuado la multiplicación gráfica $k \cdot B$, que se puede leer en el eje $k \cdot B$ dirigido hacia abajo.

En el cuadrante derecho inferior se ha efectuado finalmente la adición $A+k \cdot B$ gráficamente. El resultado a/c se puede leer en el sistema de rectas que discurren oblicuamente.

La figura 6 muestra el diagrama utilizable con clave y red auxiliar. Se utiliza como sigue:

Partiendo del valor medido $G{ }_{0}$ se dibuja una línea horizontal "1", que corta las curvas por $s_{\mathrm{z}}$ (derecha) y $s_{\mathrm{k}}$ (izquierda). Partiendo de los puntos de corte se traza una vertical ("2" y "3") hacia abajo. La vertical "2" corta la línea por $k$. Partiendo de este punto tangencial se traza una horizontal "4" hacia la derecha hasta el punto tangencial con la vertical "3" que viene de la derecha arriba. En el punto tangencial que resulta se lee el valor $\mathrm{a} / \mathrm{c}$. 
En la práctica ha resultado ventajoso inscribir en el diagrama los factores constantes dados para una receta de hormigón determinada que hay que controlar. Entonces se puede averiguar rápidamente en forma gráfica respecto de un $G_{0}$ medido el correspondiente valor a/c y leerlo (ver el siguiente ejemplo).

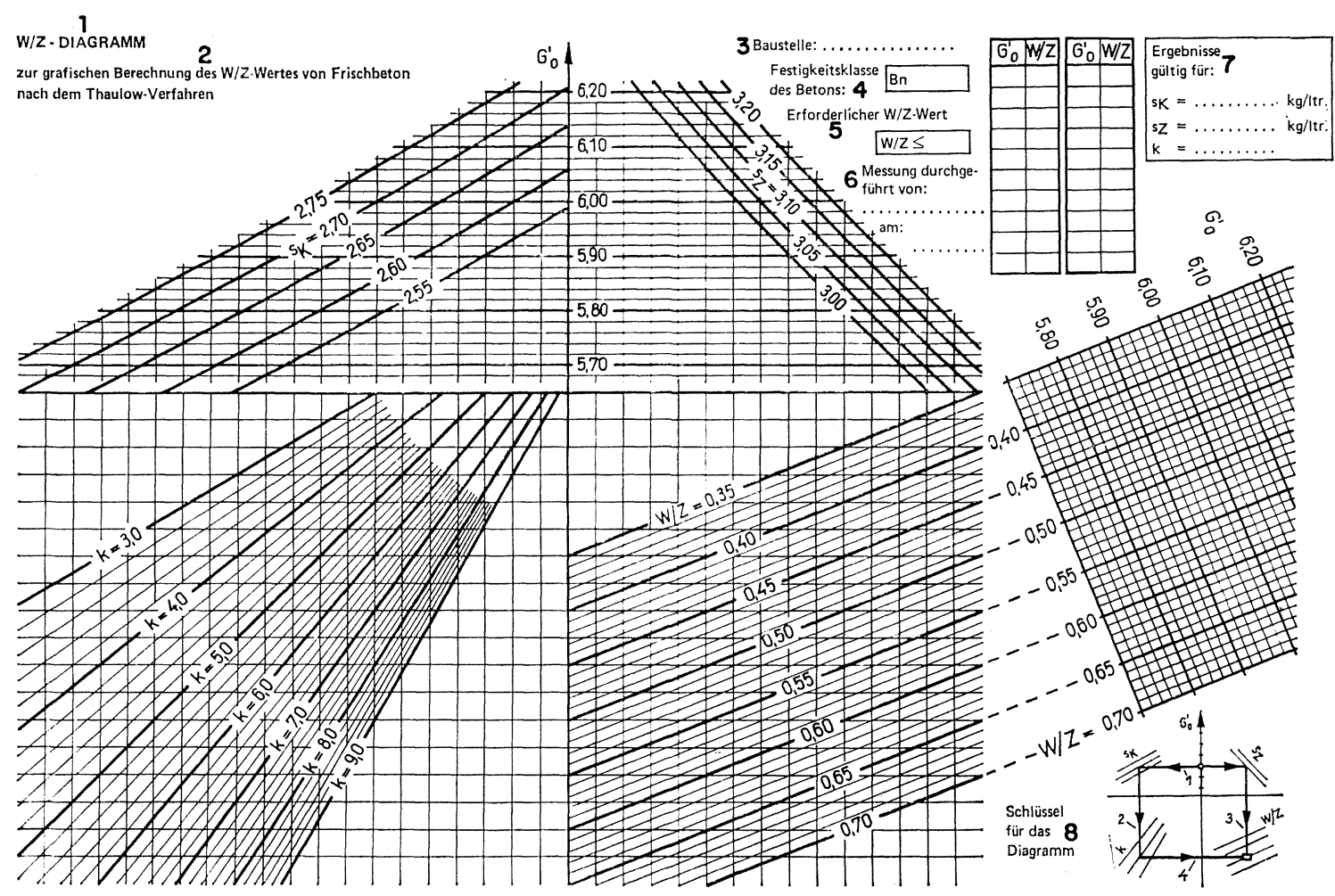

1. Diagrama agua/cemento $(a / c)$.

2. Cálculo gráfico del valor a/c del hormigón fresco según el procedimiento de Inaulow.

3. Lugar de la obra.
4. Clase de resistencia del hormigón.

5. Valor requerido $\mathrm{a} / \mathrm{c}$.

6. Evaluación efectuada.

7. Resultados válidos para:

8. Clave para el diagrama.

Fig. 6.-Diagrama a/c con clave y red auxiliar para cálculo rápido del valor a/c según el procedimiento de Thaulow.

Otra manera de facilitar la cosa, especialmente cuando se controla el hormigón en masa (por ejemplo en fábricas de transporte), consiste en efectuar de antemano la averiguación gráfica del valor a/c respecto de un valor $G^{\prime}$ bajo y elevado, de manera que se obtienen dos valores límite respecto del a/c. Los valores intermedios se pueden leer fácilmente con la red auxiliar junto al diagrama en una recta. El personal de laboratorio puede leer respecto de cualquier valor medido $G_{0}$ el correspondiente valor a/c sin volver a utilizar el diagrama a/c.

El que quiere evitar una lectura gráfica repetida en el diagrama auxiliar, utiliza el pequeño cuadro relativo a $G_{0}^{\prime}$ y a/c y registra allí el par de valores leído una vez.

Para toda nueva receta de hormigón hay que volver a utilizar naturalmente el diagrama a/c, para averiguar la relación gráfica o el cuadro entre $G_{0}^{\prime}$ y el valor a/c. El diagrama se edita por esta razón con el formato DIN A 4 como block. La figura 7 muestra un ejemplo de aplicación (10). 
Ejemplo: (figura 7).

Dada una receta de hormigón es:

$$
s_{\mathrm{z}}=3,10 ; s_{\mathrm{k}}=2,65 ; k=6,00 \text {. }
$$

Para $G_{0}$ hay que esperar o suponer como valor de medida superior 5,94, como valor de medida inferior 5,82. Del diagrama se obtiene los correspondientes valores a/c 0,43 y 0,59 . La recta a través de estos dos puntos en la red auxiliar permite leer todos los valores imaginables intermedios. Los valores prácticamente utilizables de $G_{0}^{\prime}$ y a/c fueron registrados en el cuadro. Sólo ellos volverán a utilizarse en todas las mediciones.
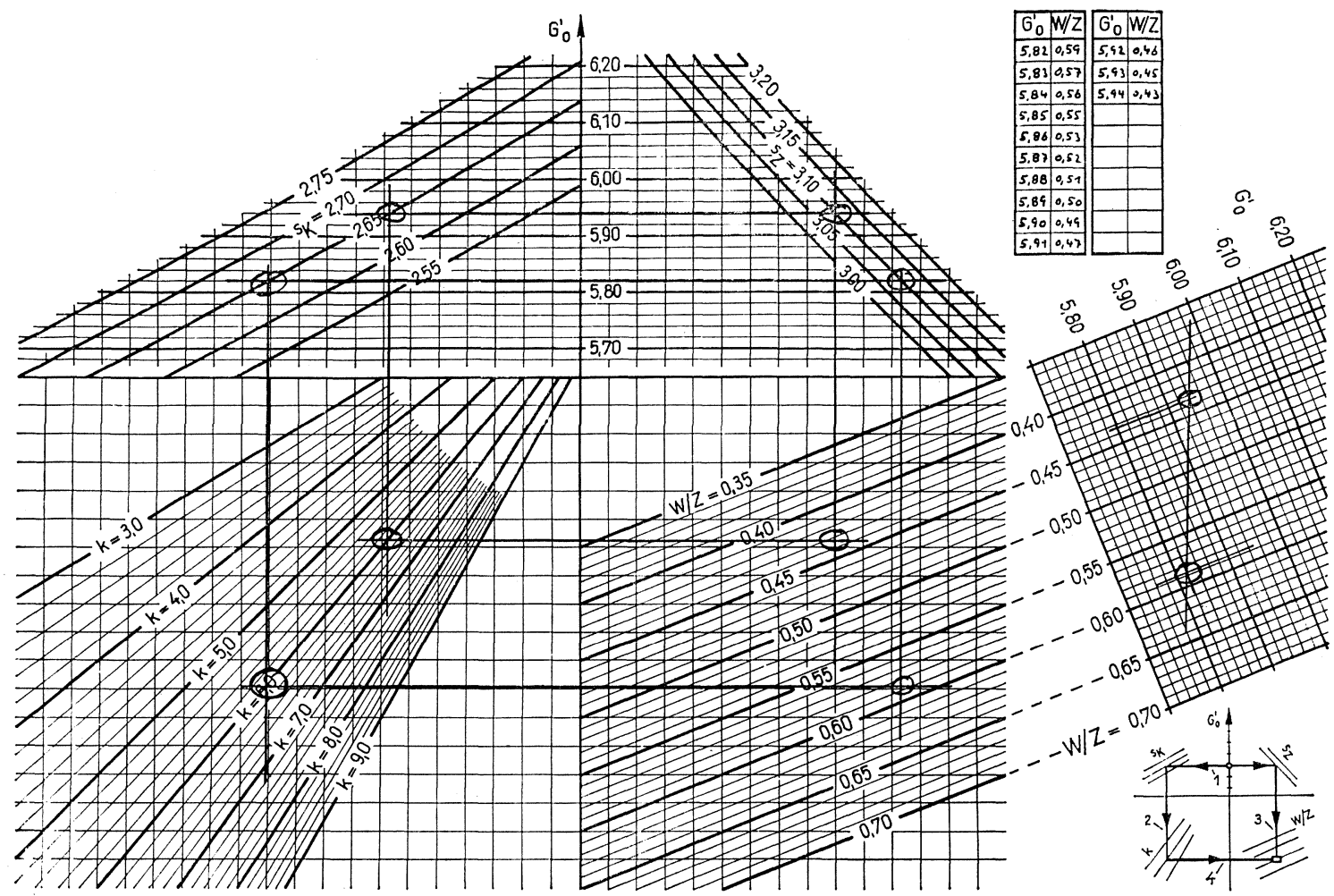

Fig. 7.-Ejemplo de la aplicación del diagrama a/c.

\section{RESULTADOS DE LAS MEDICIONES DEL VALOR a/C EN LUGARES DE OBRA Y EN FABRICAS DE HORMIGON}

La figura 8 presenta la utilización práctica de 1.999 mediciones de a/c en la red de prc babilidad. Las mediciones se efectuaron durante medio año en un gran emplazamient de la obra. Durante los trabajos de hormigonado y debido a circunstancias especiales $\mathrm{s}$ midió cada 30 minutos el valor a/c según el procedimiento Thaulow. Se trataba de u hormigón B 300 para una obra de hormigón de acero de gran amplitud. La desviació estándar llegó a 0,02 , es decir, todos los valores de medidas son de esperar práctice mente dentro de una gama de $2 \times 3 \times 0,02=0,12$, lo cual se confirma por los valores $\mathrm{d}$ medida. La fabricación del hormigón armado Tübbings (B 450) para un túnel de ferrc carril se midió en una fábrica de hormigón la distribución de los valores a/c represer tada en la figura 9 . 
En relación con un hormigón B 600 para la fabricación de traviesas de hormigón pretesado en una fábrica de hormigón resultó una distribución del valor a/c según figura 10.

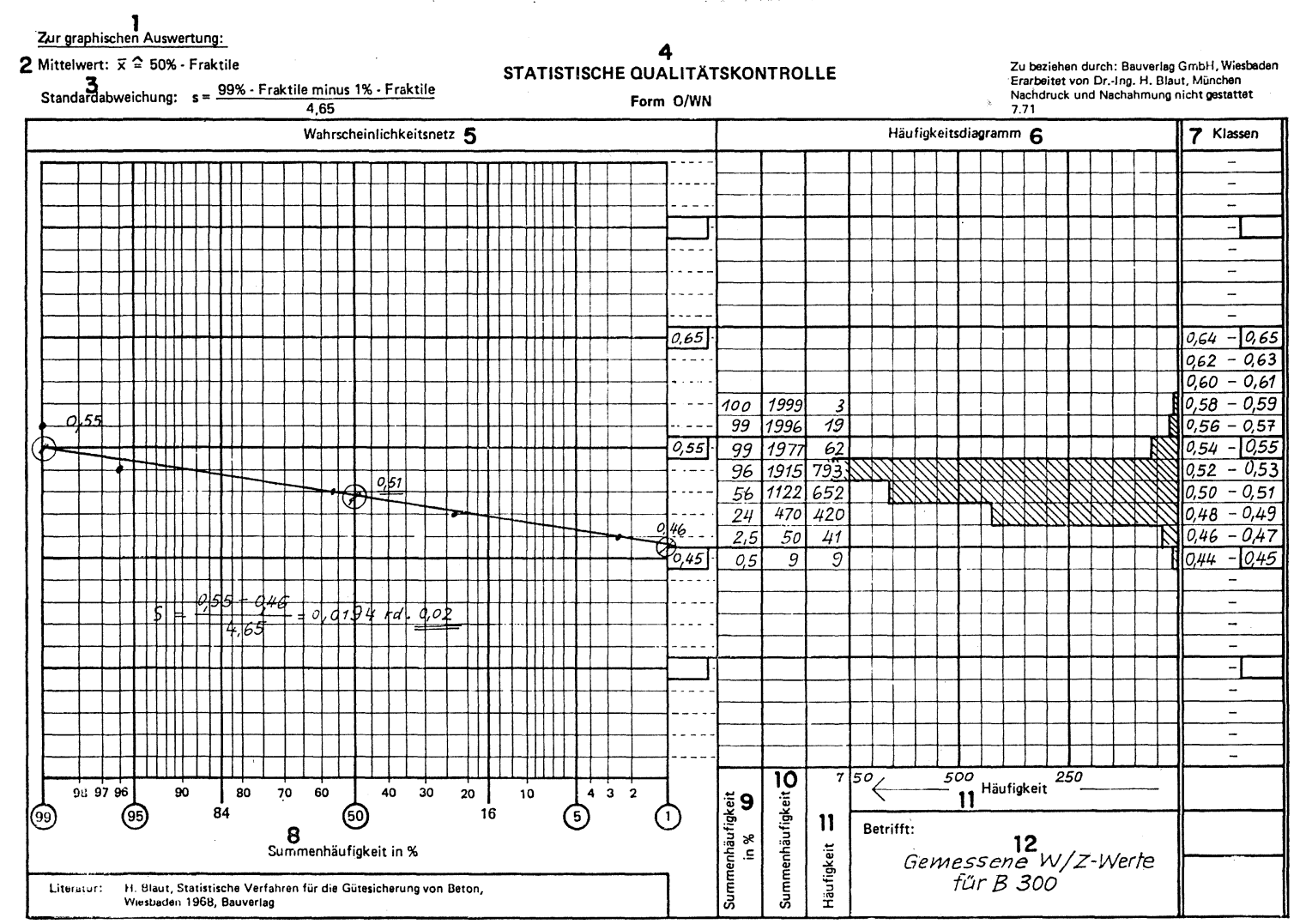

\footnotetext{
1. Cálculo gráfico.

2. Valor medido.

3. Desviación estándar.

4. Control èstadístico de calidad.

5. Red de probabilidad.

6. Diagrama de frecuencias.
}

7. Clase.

8. Desviación acumulada en \%

9. Suma de frecuencias en $\%$.

10. Suma de frecuencias.

11. Frecuencia.

12. Coeficiente $\mathrm{a} / \mathrm{c}$ de un hormigón $\mathrm{B} 300$ dilatado.

Fig. 8.-Valores a/c en la fabricación del hormigón B 300 con HOZ 275; tiempo de fabricación: aproximadamente 6 meses; número de muestras: $n=1.999$; diagrama de frecuencia y valoración en la red de probabilidad (7); valor medio: 0,51 ; deviación estándar: 0,02.

Estas obras especialmente bien controladas y los resultados obtenidos en las centrales de hormigón no pueden engañarnos respecto al hecho de que en general hay que esperar mayores dispersiones del valor a/c (y por ello también en cuanto a la resistencia del hormigón). La distribución de los valores a/c (1.179 mediciones) representada en la figura 2 corresponde a un hormigón de la calidad B 225. El valor medio es de 0,62, la desviación estándar 0,069 . La dispersión es relativamente grande y procede de un control poco eficaz del contenido de agua o de la consistencia.

Hasta que se disponga de informaciones más amplias sobre la dispersión del valor a/c debería partirse del hecho de que la desviación estándar de los valores medidos a/c a esperar en lugares de obras bien controlados o en fábricas de cemento es de 0,03 y en lugares normales de obras por lo menos de 0,05 . La dispersión de prueba de la medición del valor a/c según el procedimiento de Thaulow es según observaciones del autor del orden de 0,01 . Como por tanto es menor que el $1 / 3$ de las desviaciones estándar de los valores a/c que son de esperar, según (7) puede menospreciarse. 
1

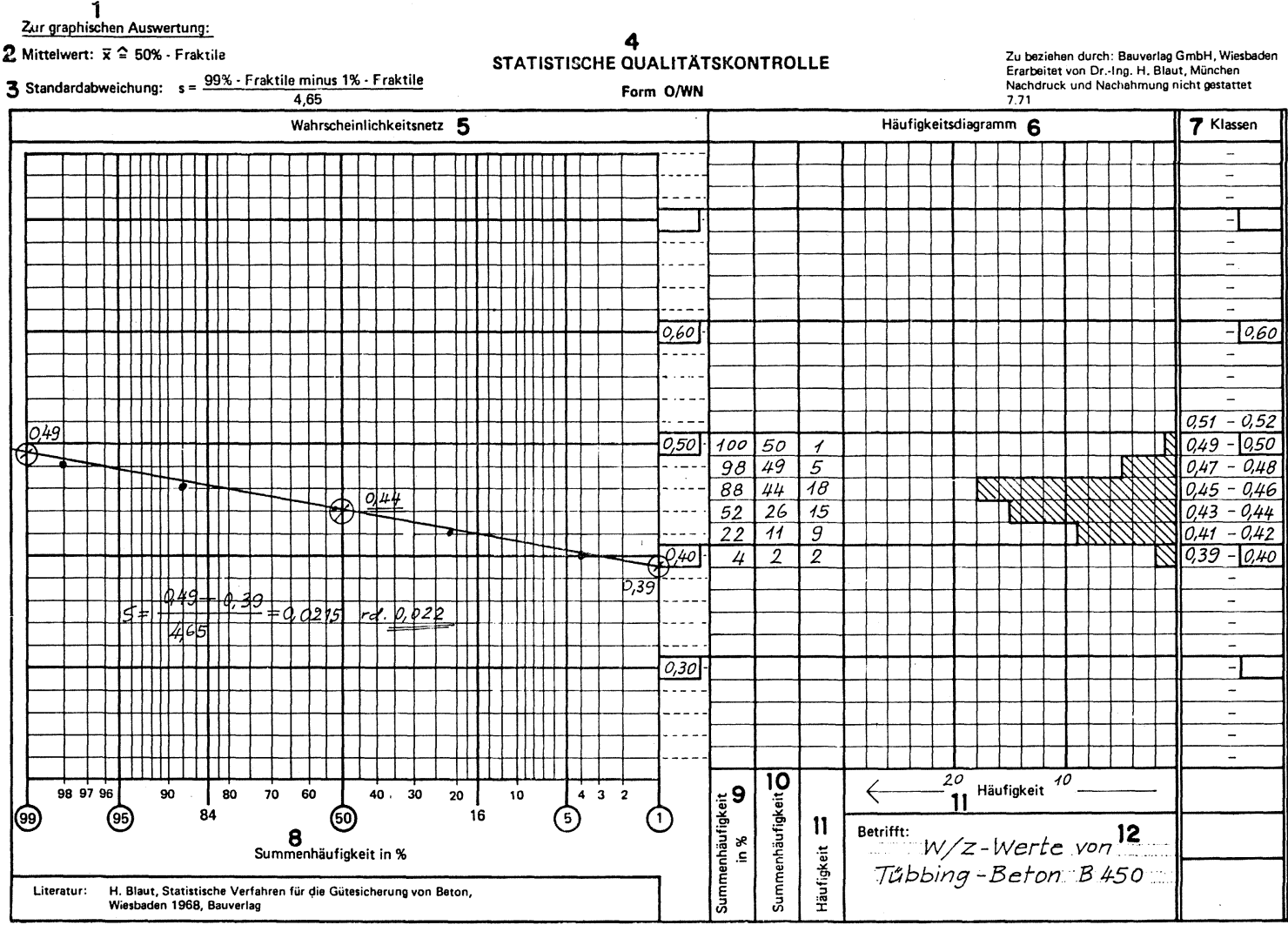

7. Clase.

8. Desviación acumulada en $\%$.

9. Desviación acumulada.

10. Suma de frecuencias.

11. Frecuencia.

12. Coeficiente $\mathrm{a} / \mathrm{c}$ de un hormigón B 450 dilatado.

5. Red de probabilidad.

6. Diagrama de frecuencias.

Fig. 9.-Valores a/c de un hormigón B 450 durante la fabricación de S-Bahn-Tübbings en una fábrica de hormigón; número de ensayos: $n=50 ; 5$ diagramas de frecuencia y valoración en la red de probabilidad (7); valor medio: 0,44 ; desviación estándar: 0,022 .

\section{RESUMEN Y OTROS DESARROLLOS}

Los resultados del presente informe se completan a continuación en forma resumida y mediante algunas consideraciones:

5.1. En la nueva norma alemana del hormigón armado DIN 1045 el valor a/c ocupa un lugar especial como valor característico de la calidad del hormigón. Con ayuda de la resistencia normativa del cemento y de los valores a/c del hormigón fresco se puede calcular la resistencia del cubo, ya durante la fabricación misma del hormigón: la medición del valor a/c permite con un gasto relativamente reducido un control útil de la producción con vistas a las propiedades deseadas del hormigón (por ejemplo resistencia).

Se dispone de un control ideal, si inmediatamente durante el mezclado del hormigón se mide la humedad real de los áridos y si puede tenerse en cuenta en la adición del agua. De acuerdo con el actual estado de la evolución esto es posible mediante una medición de la humedad de los áridos con la ayuda de fuentes de neutrones (frenado de una radiación de neutrones mediante núcleos de hidrógeno), sin embargo resulta muy costoso. Por ello el procedimiento resulta racional exclusivamente en lugares de obra muy grandes o en grandes fábricas de hormigón de transporte, que suministran un hormigón mezclado en fábrica (8). 


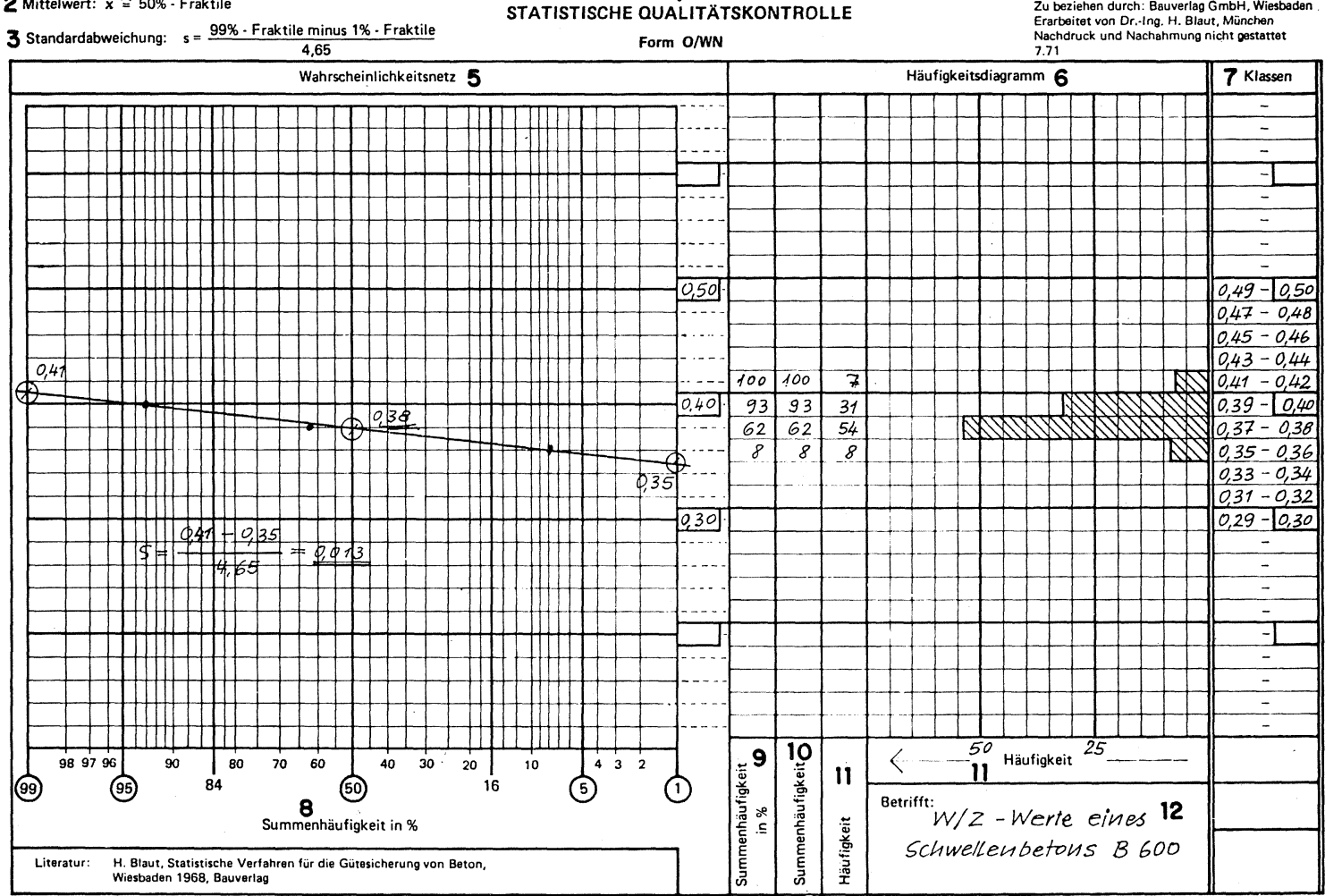
1. Cálculo gráfico.
2. Valor medio.
3. Desviación etándar.
4. Control estadístico de calidad.
5. Red de probabilidad.
6. Diagrama de frecuencias.

\author{
7. Clase \\ 8. Deviación acumulada en $\%$. \\ 9. Desviación acumulada. \\ 10. Suma de frecuencia. \\ 11. Frecuencia. \\ 12. Coeficiente $a / c$ de un hormigón B 600 dilatado.
}

Fig. 10.-Valores $a / c$ en la fabricación de traviesas de hormigón pretensado en una fábrica de hormigón en B 600 ; diagrama de frecuencia y evaluación en la red de probabilidad (7); tiempo de fabricación: 4 meses; número de pruebas: $n=100$; valor medio: 0,38 ; desviación estándar: 0,013 .

5.2. El procedimiento Thaulow permite una rápida medición del valor a/c mediante doble pesada de una muestra de hormigón fresco de $10 \mathrm{~kg}$. En este caso deben ser conocidas las densidades puras de cemento y áridos así como la proporción de mezcla de cemento y áridos.

5.3. El cálculo del valor a/c necesario después de la pesada, se puede simplificar y acelerar esencialmente mediante un diagrama auxiliar en forma del indicado "diagrama a/c (figura 6)".

5.4. Una serie de resultados de mediciones de a/c efectuadas en la obra y en la fábrica de cemento proporcionan una idea acerca del orden y dispersión del valor a/c. Estas constituyen otra confirmación de la relación existente entre el valor a/c, resistencia del cemento y resistencia del cubo o probeta.

5.5. En la evaluación de las mediciones del valor a/c para apreciar la resistencia de las probetas se supone que el hormigón está "completamente" compactado, que por consiguiente sólo quedan el $1,5 \%$ de poros de compactación que no pueden evitarse totalmente mediante la compactación habitual cuidadosa. El contenido real de poros de aire, del que depende la resistencia a la compresión, no se mide en general. 
El procedimiento de Thaulow permite averiguar el valor a/c y el contenido de poros residuales (grado de compactación) de una muestra de hormigón fresco compactado en la obra. La muestra se toma con un cilindro o en otra forma adecuada del encofrado sin perjudicarlo, una vez se pesa con aire y otra sin aire bajo el agua. Los detalles pueden tomarse de (4).

En este lugar no puede dejar de mencionarse la propuesta de Rotfuchs (9) de introducir un valor de poros/cemento (valor $\mathrm{P} / \mathrm{C}$ ) en lugar del valor a/c, por el que no sólo se tendrán en cuenta los poros del agua residual, sino también los poros de aire debidos a la defectuosa compactación y en algunos casos a los poros de aire introducidos artificialmente, prestándose atención especial a la resistencia a la presión.

5.6. A medida que van aumentando las firmas de construcción emplean "hormigón de central" en sus obras. De este modo pierden la posibilidad de influir en la calidad del hormigón directamente. El hormigón de central obliga a una división del trabajo, que tiene ventajas e inconvenientes. Ventajas, porque una central de hormigón preparado perfectamente equipada puede fabricar el hormigón con reducidos costes en la mayor parte de los casos. Inconvenientes, porque no es posible efectuar ya directamente el control de la calidad del hormigón, es decir, por medio del conductor de la hormigonera y cada una de las componentes de la mezcla en la obra, sino que debe efectuarse dando un rodeo por medio de las condiciones de suministro y el control de calidad. En este caso serviría de gran ayuda para el receptor del hormigón que al recibirse el hormigón fresco en la obra se pudiera averiguar mediante una comprobación rápida y sencilla si el hormigón tiene las propiedades acordadas en el pedido.

La medición del valor a/c representa así una cierta ayuda; sin embargo depende de algunas informaciones, que debe suministrar el fabricante de hormigón. Esto perjudica en este caso su valor como procedimiento objetivo de comprobación. El autor opina que el desarrollo de un procedimiento de comprobación, que se apoya exclusivamente en las mediciones efectuadas en el hormigón fresco, es urgentemente necesario para el futuro. E] desecado o tostación del hormigón fresco, aplicado hoy en día muchas veces para averiguar el valor a/c tampoco prescinde de las informaciones que debe suministrar el fabri. cante del hormigón; pues en la averiguación del valor a/c debe conocerse en este caso e] volumen de cemento por metro cúbico de hormigón.

Las hojas que aparecen en las figuras 9 y 10 se pueden obtener en la Bauverlag GmbH de Wiesbaden.

\section{B I B L I O G R A F I A}

(1) AbRams, D. A.: Desing of Concrete Mixtures, Chicago 1918; Bestimmung von Betonmischungen, Au torisierte Übersetzung, heraugegeben von der SIA-Fachgruppe für Beton und Eisenbetoningenieure al Mitteilung Nr. 1 der Gußbetonkommission.

(2) WaLz, K.: Herstellung von Beton nach DIN 1045, Düsseldorf 1971.

(3) Walz, K.: Anleitung für die Zusammensetzung und Herstellung von Beton mit bestimmten Eigens chaften, Berlin 1958.

(4) Thaulow, S.: Field testing of concrete, Norsk Cementforening, Oslo 1952; ein Auszug in deutsche Sprache erschien in "Betonbau des Auslandes", $\mathrm{Nr} 55 / 57$, herausgegemen vom Deutschen Beton-Ve rein, Wiesbaden 1957.

(5) Arbeitsblatt für die Prüfung von Frischbeton nach dem Thaulow-Verfahren, Liste 10/301/171 de Fa Curt Weißgerber, Frankfurt/Main.

(6) Arbeitsblatt für die Prüfung von Zuschlägen nach dem Thaulow-Verfahren, Liste 2/303/471 der Fء Curt Weissgerber, Frankfurt/Main.

(7) Blaut, H.: Statistiche Verfahren für die Gütesicherung von Beton, Wiesbaden 1968.

(8) Rozinskr, F.: Die Betonherstellung beim Donaukraftwerk Ottensheim-Wilhering, Zement und Betor 1972, Nr. 61/62.

(9) Rothfuchs, G.: Betonfibel, Band II. Wiesbaden 1964.

(10) "Formblatt" W/Z-Diagramm von Dr.-Ing. H. Blaut zur grafischen Berechnung des W/Z-Wertes vo Frischbeton nach dem Thaulow-Verfahren, Bauverlag GmbH, Wiesbaden 1972. 